\title{
Infant to staff ratios and risk of mortality in very low birthweight infants
}

\section{A Callaghan, D W Cartwright, P O'Rourke, M W Davies}

\begin{abstract}
Objectives: To assess the effect that infant to staff ratios, in the first three days of life, have on the survival to hospital discharge of very low birthweight infants (<1500 g), having adjusted for initial risk and unit workload.

Design: In a retrospective analysis of a cohort of patients, the number of infants per nurse per shift were averaged for the first three days after admission and related to risk of mortality by logistic regression analysis. Infant to staff ratio was divided into terciles of low (1.16-1.58), medium (1.591.70), and high (1.71-1.97) infants per staff member.

Subjects: 692 very low birthweight infants admitted to the Intensive Care Nursery, Royal Women's Hospital, Brisbane over a four year period from January 1996 to December 1999. Main outcome measures: Survival to hospital discharge, adjusted for initial risk using the Clinical Risk Index for Babies (CRIB) score, and adjusted for unit workload using dependency scores.

Results: There were 80 deaths among the 692 babies analysed for the study period. The odds of mortality, adjusted for initial risk and infant dependency scores (unit workload), were improved by $82 \%$ when an infant/staff ratio of greater than 1.71 occurred, suggesting improved survival with the highest infant/staff ratio. The low and medium staffing levels corresponded with similar odds ratios for mortality.

Conclusions: Infants exposed to higher infant to staff ratios have an improved adjusted risk of survival to hospital discharge.
\end{abstract}

Correspondence to: Dr M Davies, Grantley Stable Neonatal Unit, Royal Women's Hospital, Butterfield St, Herston, QLD, 4006 Australia; Mark_Davies@ health.qld.gov.au

Accepted 27 June 2002

M ortality rates in very low birthweight (VLBW) babies have been studied extensively and a number of causative factors have been elucidated. However, the impact of nursing staff levels and workload of neonatal units has not been widely investigated. A study conducted in the UK (seven Scottish and two Australian neonatal units) suggested that the risk of infant mortality is independently related to staffing levels of neonatal intensive care units, particularly to the care in the first three days of life. ${ }^{1}$ They found that risk of mortality increased as infant to staff ratios increased, with a $79 \%$ increase in odds of mortality when more than 1.7 infants were assigned per nurse per shift.

Mortality rates in Australian Neonatal Intensive Care Units (NICUs) have been found to compare favourably with those in England and Scotland, ${ }^{2}$ and it has been suggested that organisational differences in NICUs between the two countries may account for this difference in outcome. Recommended nursing staff to patient ratios are higher in Australia, as advised by the Australian Health Ministers Advisory Council (one ventilated infant per nurse as opposed to two per nurse recommended in the UK). The impact of nursing staff workload and nurse to infant ratios have not been investigated in Australian neonatal units apart from the aforementioned study which also involved units from the UK.

In this study, we aimed to investigate the association between infant to staff ratios and the outcome of VLBW birth weight babies, within one NICU at the Royal Women's Hospital, Brisbane.

\section{METHODS}

The study was a retrospective analysis of prospectively collected data pertaining to a cohort of patients of less than $1500 \mathrm{~g}$ birth weight admitted to the Royal Women's Hospital Intensive Care Nursery (ICN) between January 1996 and December 1999. The ICN provides medical and surgical intensive care to approximately 1100 infants per year and receives admissions from the central and northern suburbs of Brisbane as well as regional areas including northern New South Wales, and regional Queensland. One hundred and forty regular nursing staff (104 full time equivalents) staff the ICN, but extra requirements are filled by casual and agency staff. The nursing shifts are eight hours in length and the usual requirements for staffing are dependent upon whether patients are categorised as intensive (requiring 1:1 nurse per infant), high dependency ( $1: 2$ nurse per infant), medium dependency ( $1: 3$ nurse per infant), or recovery ( $1: 5$ nurse per infant) patients. Medical staffing in the ICN is fairly constant, with one consultant neonatologist available during ordinary hours every day, and one of six visiting medical officers or two full time staff neonatologists available on call (within 30 minutes) during evenings and nights. There are three registrars and a senior house officer available on weekdays and one registrar on weekends, evening shifts, and night duties.

Data describing infant numbers, infant characteristics related to birth history, and admission details, as well as physiological data recorded in the first 12 hours of life, are routinely recorded on a Microsoft Access database at the Royal Women's Hospital ICN. This database was accessed to extract information on all VLBW admissions from January 1996 to December 1999. Data regarding the number of nurses working per shift are collected every shift and recorded on a separate database. A database was constructed containing the patient characteristics and data on the maximum number of babies per shift, as well as staffing numbers in the intensive care nursery each shift for the 72 hours (nine shifts) following the admission of a VLBW infant to the ICN. Dependency data, categorising individual patients as intensive care (requiring l:1

Abbreviations: CRIB, clinical risk index for babies; NICU, neonatal intensive care unit; OR, odds ratio; VLBW, very low birthweight 
nurse:patient), high dependency (1:2), medium dependency $(1: 3)$, or recovery $(1: 5)$, were also collected, and the expected number of nurses for each shift calculated into a dependency score. Patient characteristics included physiological data including birth weight, gestational age, presence of congenital anomalies, maximum and minimum oxygen requirement, and worst base excess in the first 12 hours of life. The primary outcome measure was mortality before hospital discharge. Infants with an inevitably lethal congenital anomaly were excluded along with those infants who were not admitted to the ICN within 36 hours of birth. Data that were not available from the database were retrieved by chart review.

The clinical risk index for babies (CRIB) scoring system is a method for assessing initial neonatal risk in infants $<1500 \mathrm{~g}$ birth weight, or $<31$ weeks gestation, ${ }^{3}$ validated in the UK but not in Australian neonatal intensive care nurseries. The CRIB score ranges from 0 to 23; higher scores indicate increasing clinical risk and severity of illness.

CRIB scores were calculated for every infant admitted during the study period to enable adjustment of mortality risk. The CRIB scoring system was then validated for this cohort of babies and was found to have a strong correlation with mortality risk. The CRIB scores were categorised into three groups: $0-2,3-10$, and $>10$ for analysis.

\section{STATISTICS}

Statistical analysis was carried out using SPSS version 10 software. ${ }^{4}$ Descriptive data were calculated for infant to staff ratios, and CRIB scores, including mean, maximum, and minimum, in survivors and in those that died before hospital discharge. Pooled data for each infant were also calculated by summing the staff numbers and infant numbers respectively over the nine nursing shifts (72 hours) after admission and calculating the ratio of infants to staff. A pooled dependency score was calculated in a similar way, averaging the dependencies over the nine shifts for each baby, to give a measure of workload within the ICN.

The pooled infant to staff ratios were divided into deciles initially and analysed by logistic regression analysis using CRIB categories, dependency scores, and infant to staff ratios as independent variables with infant mortality before hospital discharge as the outcome. Odds ratios (OR) and confidence intervals were calculated for the 10 groups, with the first decile-that is, the group with the lowest infant to staff ratio-as the comparative group. A stepwise pattern was seen with the first 6 deciles being similar to the reference group, but with significant ORs in the last three deciles. Thus the pooled infant staff ratios were grouped into terciles to simplify presentation of the data. Place of birth was also entered as a variable in the logistic regression analysis (inborn versus outborn) but showed no significant difference.

Student's $t$ test and ANOVA were used to compare means where appropriate and $\chi^{2}$ tests to compare proportions.

\section{RESULTS}

There were 709 VLBW admissions during the study period and 17 were excluded from analysis. Of these, four were excluded due to lethal congenital malformations, and 13 were excluded due to delayed admission to the unit ( $>36$ hours after birth). The overall hospital mortality rate was $12 \%$ ( 80 out of 692 VLBW infants).

Data describing characteristics of the study population are included in table 1 . There was a statistically significant difference between the mean CRIB score of babies who died before hospital discharge and the mean CRIB score of survivors $(\mathrm{p}<0.001$; see table 2$)$. Table 3 shows the association between increasing CRIB scores and mortality within the three CRIB categories used in the logistic regression analysis $\left(\chi^{2}\right.$,
Table 1 Characteristics of study infants

\begin{tabular}{lll}
\hline Characteristic & Number \\
\hline Study infants & 692 & \\
Hospital mortality & $80(12 \%)$ & \\
Congenital malformations & $82(12 \%)$ & \\
Male & $366(53 \%)$ & \\
Outborn & $118(17 \%)$ & \\
\cline { 2 - 3 } & Median & Interquartile range \\
\cline { 2 - 3 } & 1088 & 861 to 1295 \\
Birthweight (grams) & 28.4 & 26.9 to 30.1 \\
Gestation (weeks) & 6 & 4 to 8 \\
APGAR 1 minute & 8 & 7 to 9 \\
APGAR 5 minute & 0.50 & 0.27 to 0.80 \\
Max $\mathrm{O}_{2}$ requirement $\left(\mathrm{FiO}_{2}\right)$ & 0.21 & 0.21 to 0.28 \\
Min $\mathrm{O}_{2}$ requirement $\left(\mathrm{FiO}_{2}\right)$ & -4.0 & -6.3 to -1.9 \\
Base excess & 20.8 & 19.1 to 23.6 \\
No infants/shift & 12.8 & 12.1 to 13.7 \\
No staff/shift & 16.9 & 15.0 to 19.4 \\
Dependency & & \\
\hline & &
\end{tabular}

Table 2 The association between outcome and mean CRIB scores

\begin{tabular}{llll}
\hline Outcome & $\mathrm{N}$ & $\begin{array}{l}\text { Mean CRIB } \\
\text { score (SD) }\end{array}$ & $\begin{array}{l}\text { Mean pooled infant:staff } \\
\text { ratio (SD) }\end{array}$ \\
\hline Died & 60 & $9.98(4.47)$ & $1.61(0.11)$ \\
Survived & 612 & $\begin{array}{l}3.18(3.21) \\
\mathrm{p}<0.001\end{array}$ & $\begin{array}{l}1.65(0.13) \\
\mathrm{p}=0.025\end{array}$ \\
Test & 692 & $3.97(4.01)$ & $1.64(0.13)$ \\
\hline
\end{tabular}

\begin{tabular}{lllll} 
Table 3 & \multicolumn{2}{l}{ Outcome by CRIB categories } \\
\hline & \multicolumn{2}{l}{ CRIB category } & \\
\cline { 2 - 4 } Outcome & $0-2$ & $3-10$ & $11-23$ & Total \\
\hline Survived & 387 & 206 & 19 & 612 \\
$\begin{array}{l}\text { Died (\% of total) } \\
\text { Total }\end{array}$ & $5(1.2 \%)$ & $37(15 \%)$ & $38(67 \%)$ & 80 \\
& 392 & 243 & 57 & 692 \\
\hline
\end{tabular}

$\mathrm{p}<0.0001$ ). These results validate the use of the CRIB scoring system as a tool for calculating adjusted mortality risk in this population of babies.

The $t$ test for equality of means revealed that the mean pooled infant to staff ratio in surviving infants (1.65) was significantly different from the mean in those who died before hospital discharge (1.61; see table 2).

The characteristics of the three infant to staff ratio groups (terciles) are shown in table 4 . There was no significant difference between the mean CRIB scores in the three groups (ANOVA $p=0.137$ ). The logistic regression analysis for mortality before hospital discharge revealed a statistically significant result for the highest tercile of infant to staff ratio. The crude odds ratios (OR), the OR adjusted for CRIB score and the OR adjusted for both CRIB and dependency score all revealed a significant reduction in mortality risk. The low and medium staffing levels had similar mortality levels in all analyses.

\section{DISCUSSION}

A number of studies in adult intensive care units have shown an increased risk of mortality and morbidity associated with increased nursing workload. ${ }^{5}$ Other studies outside of intensive care show a similar association between morbidity and 
Table 4 Outcome by infant:staff ratios, pooled over 9 shifts (divided into terciles) with crude odds ratio (OR) of mortality and adjusted mortality OR (adjusted for CRIB only, CRIB, and dependency)

\begin{tabular}{llllllll}
\hline $\begin{array}{l}\text { Infant:staff pooled } \\
\text { ratios }\end{array}$ & $\begin{array}{l}\text { CRIB mean } \\
\text { (SD) }\end{array}$ & Survived & $\begin{array}{l}\text { Died } \\
\text { (\% of total) }\end{array}$ & Total & Crude OR (95\% CI) & $\begin{array}{l}\text { Adjusted OR } \\
\text { (95\% CI) (CRIB) }\end{array}$ & $\begin{array}{l}\text { Adjusted OR (95\% CI) } \\
\text { (CRIB \& Dependency) }\end{array}$ \\
\hline $1.16-1.58$ (low) & $3.79(4.05)$ & 202 & $30(12.9)$ & 232 & & & \\
$1.59-1.70$ & $4.40(4.27)$ & 193 & $37(16.1)$ & 230 & $1.29(0.77$ to 2.17$)$ & $1.05(0.55$ to 2.00$)$ & $0.84(0.42$ to 1.66$)$ \\
$1.71-1.97$ & $3.71(3.69)$ & 217 & $13(5.7)$ & 230 & $0.40(0.21$ to 0.80$)$ & $0.32(0.15$ to 0.71$)$ & $0.18(0.06$ to 0.5$)$ \\
\hline
\end{tabular}

workload. ${ }^{78}$ Iatrogenic complications are more common in units with higher patient to staff ratios and higher workloads. ${ }^{6}$ Explanations for this include decreased surveillance for errors ${ }^{69}$ as well as variable levels of skills among nursing staff.

Hamilton et al suggested a similar association between mortality and infant:staff ratios in neonatal nurseries. ${ }^{1}$ However, our data, with a much larger patient population, suggest that an increase in the patient to staff ratio does not increase the risk of mortality. When comparing with the lowest patient to nurse ratio, the odds of mortality in the highest infant to staff ratio are actually reduced $(\mathrm{OR}=0.18)$.

Place of birth of the study infants was considered to be a possible confounding variable. Seventeen per cent of the infants involved were born outside of the RWH ICN. Although babies are retrieved quickly after birth, some regional areas of Queensland are a long distance from Brisbane and the retrieval process in itself can be quite time consuming. Although their data from the first 12 hours after birth reflect their risk of mortality, studies suggest that outborn infants have a poorer outcome compared with those born at a tertiary NICU. ${ }^{10}{ }^{11}$ When statistical analysis was carried out on inborn babies alone, similar results were seen, and when outborn status was included as a variable in analysis it did not reach statistical significance nor have an impact on other factors in the model.

The CRIB scoring system is now widely utilised and has been validated as being more accurate than birth weight or gestational age in assessing initial risk of hospital mortality among infants admitted to neonatal intensive care. ${ }^{12-14}$ We have shown in the sample of babies in this study that CRIB scores reliably predict mortality. Mean CRIB scores are higher in those infants that died (see table 2), and mortality is higher in babies with higher CRIB scores (see table 3). However, this scoring system has some limitations. The scoring of congenital anomalies is subjective (lethal, acutely life threatening, not acutely life threatening, none) and does not take into account the severity of a lesion, however we excluded only four babies with lethal congenital anomalies.

A possible explanation for the reduction in mortality among the highest infant:staff ratio is that increased handling of small, unstable infants may occur when there are more nursing staff available to perform care. It is possible that the interventions that we consider beneficial, such as frequent suctioning and turning, may in fact be detrimental if carried out too frequently.

Other possible explanations for this include our inability from this data to take into account medical staff presence within the ICN, or to measure nursing workload and individual patient workload accurately, and varying levels of nursing staff skills. We attempted to carry out a measure of workload using patient dependencies as a variable. This adjustment for dependencies intensified the measured effect rather than explaining it, as would be expected of a confounder.

Actual nursing workload is often measured in adult studies, ${ }^{15} 5$ as well as patient to staff ratios, using various workload measures such as subjective assessments, or more formalised scoring systems such as TISS ${ }^{16}$ and GRASP. ${ }^{17}$ It has been shown that increased workload within a unit is an independent risk factor for risk of mortality, with an increase of $210 \%$ in the odds ratios for mortality for patients exposed to high ICU workload over those exposed to moderate ICU workload. ${ }^{5}$ Our results, suggesting that those infants exposed to higher infant:staff ratios have a lower mortality risk, suggest the opposite in VLBW infants. By categorising patients into intensive, high dependency, medium dependency, or recovery (the categories used to determine nursing staff numbers in the ICN) we broadly accounted for workload. However, there is potentially a large variability between an infants' workload with regard to medications, feeding regimens, ventilation requirements, and other factors that are not taken into account in our study.

The staffing numbers do not reflect the number of permanent nurses compared with agency staff per shift. Generally, when the nursery is quiet, staffing is entirely by specialised nurses, however when the number of babies increases, there is often a requirement for casual and agency staff who are not all trained in caring for level three neonatal intensive care babies. Studies have shown that there is an association between nursing specialisation and decreased mortality rates. ${ }^{18}$ Although in our unit the less experienced/less specialised staff usually care for the less sick infants, they are often involved in assisting in care for the sicker infants during meal breaks and when two staff members are required for a particular duty.

Our mortality rates are much lower than those reported in the $\mathrm{UK}^{23}$ and it has been suggested that differences in staffing may reflect this. Certainly our staffing levels did not vary greatly, with the pooled staffing ratio being very similar to the maximum infant to staff ratio. Studies have also suggested a lower mortality rate in tertiary units with a high volume of patients..$^{11}$

The results of this study are thought provoking, and suggest the need for further investigation into the role of infant:staff ratios as an independent risk factor in the mortality of VLBW babies. A prospective study, looking at both total infant and staff numbers as well as individual nursing workloads and perceived staffing requirements versus actual staffing supplied may clarify the issue of the effect of staffing on infant mortality. The number of permanent staff compared with agency and casual staff and the impact of medical staffing should also be taken into account in further studies.

\section{ACKNOWLEDGEMENTS}

The authors wish to thank Sharon Sanders, Public Health Program, Medical School, Herston Rd, Herston Queensland 4006, Australia.

\section{Authors' affiliations}

L A Callaghan, D W Cartwright, M W Davies, Grantley Stable Neonatal Unit, Royal Women's Hospital, Brisbane, Australia M W Davies, Perinatal Research Centre, and Department of Paediatrics and Child Health, The University of Queensland, Brisbane, Australia P O'Rourke, Department of Social and Preventive Medicine, The University of Queensland, Brisbane, Australia

L A Callaghan, Department of Paediatrics, Caboolture Hospital, Caboolture, Australia 


\section{REFERENCES}

1 Hamilton K, Gould C, Tarnow-Mordi W, et al. Hospital mortality in relation to staffing levels in the first three days of neonatal care. Proceedings of the 4th Annual Congress of the Perinatal Society of Australia and New Zealand 2000. Brisbane, Australia. Sydney: PSANZ, 2000:109.

2 International Neonatal Network, Scottish Neonatal Consultants, Nurses Collaborative Study Group. Risk adjusted and population based studies of the outcome for high risk infants in Scotland and Australia. Arch Dis Child Fetal Neonatal Ed 2000;82:F1 18-23.

3 International Neonatal Network. The CRIB (clinical risk index for babies) score in auditing the performance of neonatal intensive care units. Lancet 1993:342:193-8.

4 SPSS. Statistical Package for the Social Sciences. SPSS for Windows, Version 10, Chicago: SPSS Inc, 1998.

5 Tarnow-Mordi WO, Hau C, Warden A, et al. Hospital mortality in relation to staff workload: four year study in an adult intensive-care unit. Lancet 2000:356:185-89.

6 Giraud T, Dhainaut JF, Vaxelaire JF, et al. latrogenic complications in adult intensive care units. A prospective two-center study. Crit Care Med 1993;21:40-51.

7 Shamian J, Hagen B, Hu T, et al. The relationship between length of stay and required nursing hours. J Nurs Admin 1994;24:52

8 Flood SD, Diers D. Nurse staffing, patient outcome, and cost. Nurs Management 1988;19:34.
9 Donchin $Y$ Gopher D, Olin M, et al. A look into the nature and causes of human errors in the intensive care unit. Crit Care Med 1995;23:294-300.

10 Phibbs CS, Bronstein JM, Buxton E, et al. The effects of patient volume and level of care at the hospital of birth on neonatal mortality. JAMA 1996;276:1054-9.

11 Paneth N, Kiely J, Wallenstein S, et al. The choice of place of delivery: effect of hospital level on mortality on all singleton births in New York City. Am J Dis Child 1987;141:60-4

12 Rautonen J, Makela A, Boyd H, et al. CRIB and SNAP: assessing the risk for preterm neonates. Lancet 1993;343:1272-3.

13 De Courcy Wheeler RH, Wolfe CDA, Fitzgerald A, et al. Use of the CRIB (clinical risk index for babies)score: a tool for assessing initial neonatal risk of mortality and morbidity. Arch Dis Child Fetal Neonatal Ed 1996;75:F49-52.

14 Baumer JH, Wright D, Mill T. Illness severity as measured by CRIB score: a product of changes in perinatal care? Arch Dis Child Fetal Neonatal Ed 1997:77:F211-15.

15 Byrick RJ, Caskennette GM. Audit of critical care: aims, uses, costs and limitations of a Canadian system. Can J Anaesth 1992;39:260-9.

16 Keene AE, Cullen DJ. Therapeutic intervention scoring system: update 1983. Crit Care Med 1983;11:1-3.

17 Meyers D. GRASP, a patient information and workload management system. Morgantown, NC, MCSI, 1978.

18 Czaplinski C, Diers D. The effect of staff nursing on length of stay and mortality. Med Care 1998;36:1626-38.

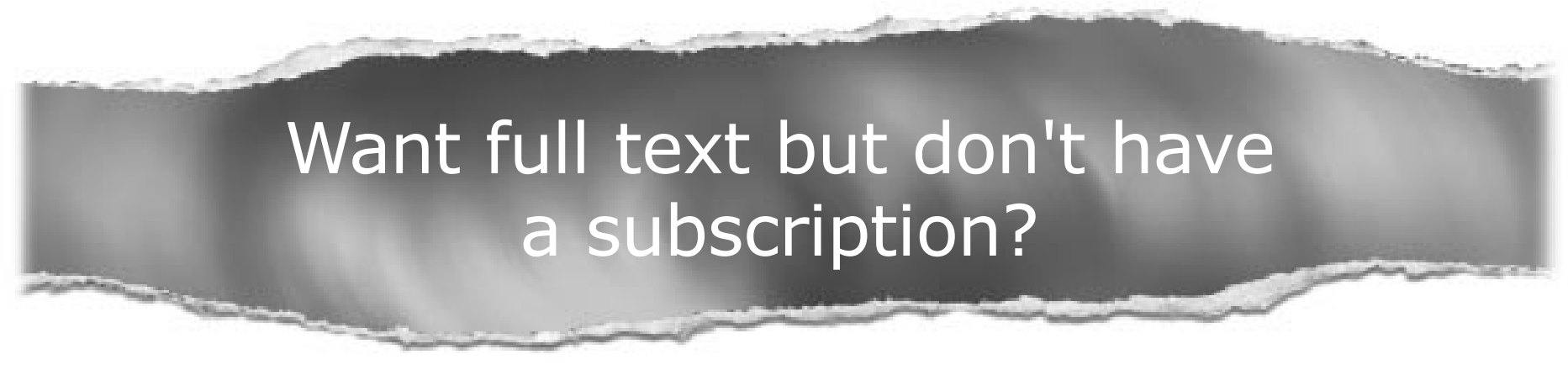

Pay per view

For just $\$ 8$ you can purchase the full text of individual articles using our secure online ordering service. You will have access to the full text of the relevant article for 48 hours during which time you may download and print the pdf file for personal use.

\section{www.archdischild.com}

